KENTRON

REVUE PLURIDISCIPLINAIR

DU MONDE ANTIQUE

\section{Kentron}

Revue pluridisciplinaire du monde antique

22 | 2006

La démesure (suite 2)

\title{
Emendatiunculae Malaterrenae
}

\section{Olivier Desbordes}

\section{OpenEdition}

Journals

Édition électronique

URL : http://journals.openedition.org/kentron/1780

DOI : 10.4000/kentron. 1780

ISSN : 2264-1459

\section{Éditeur}

Presses universitaires de Caen

\section{Édition imprimée}

Date de publication : 31 décembre 2006

Pagination : 199-208

ISBN : 2-84133-296-0

ISSN : 0765-0590

Référence électronique

Olivier Desbordes, « Emendatiunculae Malaterrenae», Kentron [En ligne], 22 | 2006, mis en ligne le 21 mars 2018, consulté le 22 novembre 2020. URL : http://journals.openedition.org/kentron/1780 ; DOI : https://doi.org/10.4000/kentron.1780

\section{@ $\oplus \Theta \Theta$}

Kentron is licensed under a Creative Commons Attribution-NonCommercial-NoDerivatives 3.0 International License. 


\section{EMENDATIUNCULAE MALATERRENAE ${ }^{1}$}

Le De rebus gestis Rogerii [...] et Roberti Guiscardi de Geoffroi Malaterra est introduit par deux épîtres dédicatoires ${ }^{2}$ dont les formules de salutatio ont déconcerté passablement critiques et traducteurs. En voici le texte, tel qu'il a été constitué par Ernesto Pontieri pour l'édition qu'il a donnée de cette chronique dans le nouveau Muratori:

(p. 3, 2-5) Reuerendissimo memoriae nostrae Angerio, Cathanensium episcopo, frater Gaufredus, ab antecessoribus Malaterra agnomen trahens, infelici cursu mundano cum Martha habito, ad felicitatem quietis Mariae cum Lazaro fratre resuscitaui.

reuerendissimo om. $Z \|$ memoriae nostrae] beatae memoriae $A Z \|$ angerio $A$ : egregio $Z^{1}$ georgio aut gregorio $Z^{2}$ dubitanter $\|$ cathanensium] cathaniensium $A$ catheniẽn $Z$ cathanensi $e d$. pr. cathanientium $M e \|$ agnomen $A$ : agonem $Z \|$ infelici $Z^{2}$, edd. : felici $A Z^{1} \|$ fratre add. $Z^{p c} \|$ resuscitaui] -tari $A Z$-tatus $D^{\prime}$ Angelo

(p. 4, 1 sq.) Omnibus quibus per uniuersam Siciliam episcopale uel clericale nomen assignatur frater Gaufredus Malaterra cum assignatione nominis et significatum.

episcopale... clericale $A$ : -lem... -lem $Z \|$ frater om. $Z$, ed. pr. $\|$ gaufredus $Z$ : goffridus $A \|$ et $Z$ : etiam $A \|$ significatum $A Z$ : -tus Avenel D'Angelo

Ainsi felici, transmis par les deux témoins qui ont conservé le texte de ces deux pièces liminaires ${ }^{3}$, a disparu au profit de son contraire, infelici ${ }^{4}$, qui a paru plus

1. Nous exprimons notre amicale reconnaissance à M.-A. Avenel pour sa relecture critique de ces trois notes.

2. Sur l'impropriété de cette dénomination, cf. Simon 1959-1960, 136, n. 2: «Es handelt sich um ein Geleitbrief zu dem Werk, in dem der Adressat um Empfehlung der Schrift bei dem wirklichen Empfänger des Werkes gebeten wird».

3. L'accord de ces deux manuscrits, de famille différente, suffit pour faire admettre felici. Pour connaitre les principes à suivre dans l'établissement du texte, voir Resta 1964, résumé par Desbordes 2005, 111-113. $\mathrm{B}$ et $\mathrm{C}$ font matériellement défaut : le texte du premier commence avec le chapitre I de la chronique ; dans $C$, qui a été transcrit d'un «vetustissimo codice manu scripto ac dilacerato », le texte conservé débute par les mots se agere posse cognosceret $(=\mathrm{I} 2[\mathrm{p} .7,16])$. A lui-même a subi, du fait de la perte d'un des bifeuillets constituant le premier cahier, une mutilation entraînant la disparition du texte de la seconde lettre dédicatoire à partir de enerui poe] tria quasi lacum profundissimum eqs. - disparition fâcheuse qui, en l'absence de C, prive l'éditeur du contrôle de la meilleure des deux recensions du texte.

4. Cet infelici, qui ne se voit opposer dans l'apparat de Pontieri aucune variante manuscrite, est une conjecture de Zurita $\left(=\mathrm{Z}^{2}\right)$. 
naturel à tous les éditeurs jusqu'à M.-A. Avenel exclusivement - au nom sans doute du primat donné traditionnellement à la vie contemplative sur la vie active ${ }^{5}$. D'autre part, en dépit ici encore de l'unanimité de la tradition - manuscrite et imprimée -, on a récusé l'autorité de resuscitari pour substituer à l'infinitif réputé inconstructible qui le personnel resuscitaui, qui le participe resuscitatus. Aucun de ces deux arrangements n'a été motivé expressément. Le premier, indiqué par Pontieri, est simplement enregistré dans l'apparat de son édition ${ }^{6}$; quant au resuscitatus avancé par E. D'Angelo, on ne lui trouvera guère d'autre justification que celle qui découle de la position que ce savant lui a assignée dans le tableau des concordances qu'il s'est cru fondé à établir entre les unités constitutives des deux salutationes, lequel lui permet de conclure à une "bipartizione basata su un dativo seguito da un nominativo, senza verbo ${ }^{7}$. Seulement cette division bipartite de la salutatio ne se conforme pas aux règles qui, à cette époque, président à sa composition. Apparaît en effet ordinairement en première place, au datif, le nom du destinataire de la lettre (inscriptio), suivi du nom, au nominatif, de l'expéditeur (intitulatio). Ce renversement de l'ordre canonique, dicté par l'humilité, n'apparaît selon toute vraisemblance dans l'épistolographie de langue latine qu'avec certains auteurs chrétiens du IV ${ }^{\mathrm{e}}$ siècle $^{8}$. Mais la

5. Voir, dans Lc 10, 38-42, l'épisode fort connu de Marthe et Marie qui, ayant mis en contraste l'agitation empressée de l'une avec l'attitude paisible et attentive de l'autre, se conclut sur ces termes: [...] Maria optimam partem elegit, quae non auferetur ab ea («Marie a choisi la meilleure part [...]»).

6. «Correggo in fratre resuscitavi il fratri resuscitari di $\mathrm{P}$ [= ed. pr.] e di A » (p. 3, apparat, l. 7).

7. Cf. D'Angelo 2003, 139. Celui-ci oppose en effet d'une part les datifs Reuerendissimo [...] Angerio [...] episcopo et Omnibus [...], d'autre part les nominatifs frater Gaufredus [...] trahens [...] resuscitatus et frater Gaufredus [...] significatus. Sans doute ce découpage de l'une et l'autre salutatio définit-il des segments de texte qui ne sont pas sans analogie; mais les participes resuscitatus et significatus sont unis par une symétrie illusoire, significatus provenant d'une correction conjecturale de la leçon commune de A et de Z. Pontieri avait imprimé tel quel le texte traditionnel, sans en pénétrer le sens, considérant que «il periodo resta sospeso e non ha senso ». La retouche grammaticale introduite - mais sans explication - par M.-A. Avenel pour rétablir la construction n’améliore la phrase qu'en apparence. Il était imprudent d'appuyer une correction sur une autre correction, celle-ci apparût-elle une «ipotesi ragionevole e possibile» (D’Angelo 2003, 139).

8. Auparavant, les exceptions sont très rares: on ne cite guère que le poète Martial adressant à l'empereur Domitien la lettre dédicatoire du livre 8 de ses Épigrammes. Cyprien ou Ambroise ne dérogent pas, ou peu s'en faut, à l'usage ancien. Mais chez un Augustin, chez un Paulin de Nole, l'ordre de modestie prévaut quasi invariablement. À en juger d'après Ausone, epist. 18, 1 sq., ce nouvel ordre de préséance contreviendrait aux usages de la correspondance privée: Paulino Ausonius: metrum sic suasit ut esses / tu prior et nomen praegrederere meum. Les lettres de saint Jérôme sont, en règle générale, précédées de la seule inscriptio, d'où le datif a disparu au profit de $a d+\mathrm{acc}$. Au VI $\mathrm{VI}^{\mathrm{e}}$ siècle, chez Ennode de Pavie, les deux ordres alternent, mais à l'avantage de l'ordre ancien (77\%); dans les Variae de Cassiodore, cet ordre ancien cède le pas systématiquement à l'ordre de modestie. Sur les règles édictées en matière de préséance par les artes dictandi des XI-XII ${ }^{\mathrm{e}}$ siècles, voir par exemple Bütow 1908, 60-62. 
formule d'ouverture de nos deux épîtres dédicatoires comporte également un souhait ${ }^{9}$ - correspondant à l'antique salutatio ${ }^{10}$-, qui revêt ici d'une part la forme d'un infinitif subordonné mais en apparence indépendant, sans sujet exprimé (resuscitari), d'autre part celle d'un accusatif (significatum), l'une et l'autre forme laissant à chaque fois au lecteur le soin de suppléer le verbe requis par le sens ${ }^{11}$. Faute de connaître l'usage, la critique a donc vu des difficultés là où il n'y en avait pas. En voulant donner à la phrase de Malaterra une forme syntaxique conventionnelle et aisément intelligible, elle a dénaturé les formules de salutatio et induit de fausses interprétations. C'est ainsi que tous les traducteurs contemporains, qui ont admis les lectures de Pontieri comme le bon texte, sont partis de l'idée que Malaterra parlait de luimême ${ }^{12}$. Était-il pourtant bienséant, quand on sollicite un haut dignitaire pour qu'il vous introduise auprès du prince commanditaire de votre œuvre et qu'il vous garantisse, par son prestige, de la malveillance de détracteurs éventuels, de prétendre l'intéresser à votre petite personne par des confidences ${ }^{13}$ ? À notre connaissance, il n'est pas

9. Cf. Simon 1959-1960, 142: «Seit dem 9. / 10. Jh. hat sich eine Hauptform herausgebildet, von der fast nie mehr abgewichen wird, mit vielfach variierten Attributen $z u$ Inscriptio und Intitulatio, und mit einem Gruss verbunden [...].»

10. Sur l'évolution de la salutatio, " the most flexible part of the opening formula », voir Lanham 1975, 12; $38-54$.

11. Les artes dictandi contemporaines, qui ont discriminé soigneusement les salutationes, en proposent de plusieurs sortes : cf. Bütow 1908, 64; 66. Le verbe à suppléer ici se lit par exemple dans cet en-tête d'une lettre dédicatoire du XI ${ }^{\mathrm{e}}$ siècle: Domino meo [...] et patri Theodino abbati et omni sanctae congregationi uestrae [...] amicus uester proprius et omnium christicolarum seruus perpetuam uobis in Christo optat salutem (MGH SRM, t. IV, p. 160). Lanham 1975, 42 sq., s'est interrogée sur le pourquoi de cette ellipse du verbum finitum: "The finite governing verb goes, because it is expendable. It is not necessary for sense or style and so like a verbum dicendi it can undergo ellipsis because the infinitive proposition (or the accusative phrase, for that matter) that remains is, so to speak, its representative».

12. Le texte de Pontieri s'est imposé aux derniers traducteurs sans discussion, fût-ce sur un point de détail : «Ho percorso con Maria l’infelice strada del mondo; sono resuscitato con Lazzaro alla felicità della quiete di Maria» (Oldoni 1977, 167); «Io frate Goffredo della famiglia denominata Malaterra, dopo avere dedicato una parte dell'esistenza alle sterili cure mondane, sono rinato a nuova vita assaporando la gioia del raccoglimento spirituale» (Lo Curto 2002, 21) ; «I, brother Geoffrey, inheriting the cognomen Malaterra from my ancestors, have been reawakened from an unhappy course in the worldly condition of Martha to the felicity of the peace of Mary along with her brother Lazarus » (Wolf 2005, 41). Nous n'avons pu nous procurer la traduction d'E. Spinnato (Palerme, Flaccovio, 2000). Voici comment deux de ces traducteurs résument l'interprétation traditionnelle de cette première salutatio: «E' diventata questa, ai nostri occhi, la storia segreta d'un (sic) vita difficile, e Malaterra un uomo inquieto che ha cercato nel chiostro la soluzione a quali misteriose e personali vicende » (Oldoni $1977,167)$; «In other words, Geoffrey had been a member of the secular clergy but later became a monk» (Wolf 2005, 41).

13. C'est sans doute cette considération qui a entraîné M.-A. Avenel à écourter la formule de salutatio en incorporant au texte de la lettre la salutatio proprement dite. Mais il est singulier qu'elle n'ait pas rétabli dans sa version française le salutem implicite que sa nouvelle organisation du morceau liminaire lui imposait. De tels en-têtes ne sont plus de mise après le IX ${ }^{e}$ siècle : cf. Simon 1959-1960, 141 sq. 
d'exemple que, pour parler de soi dans le cadre restreint de l'intitulatio, un auteur ait eu recours à un autre langage que celui de l'humilité, et encore en des termes convenus, consacrés par la tradition. De fait Malaterra, ici, se nomme simplement, sans même s'attarder à qualifier son état ${ }^{14}$, et c'est un contresens que de lui appliquer ce qu'il réserve à son destinataire. Loin de faire un retour sur soi-même, il formule, en usant de métaphores qui lui sont familières ${ }^{15}$, le souhait qu'après une existence bien remplie, partagée entre l'action et la contemplation ${ }^{16}$, l'évêque de Catane gagne son salut.

On ne peut soupçonner non plus l'intégrité du texte de la seconde salutatio. Faute d'en connaître les règles, on a conclu prématurément que, telle qu'elle avait été transmise, elle devait souffrir de quelque lacune, et qu'il était impossible à tout le moins d'en reconstituer le sens intégral ${ }^{17}$. En réalité, l'accusatif significatum est, ici également, le régime d'un verbum finitum sous-entendu. Quant à l'ablatif prépositionnel cum assignatione nominis, loin de relever de «la questione del cognome Malaterra ${ }^{18}$, c'est-à-dire, en d'autres termes, de l'intitulatio, il constitue avec cet accusatif la salutatio proprement dite, laquelle, cette fois encore, énonce un souhait. Néanmoins ce morceau de texte, dont la lecture ou la construction ne sont pas douteuses, soulève une difficulté d'interprétation. Il semble que Malaterra, s'adressant maintenant à l'ensemble des évêques et des clercs de Sicile, veuille les mettre en garde contre la tentation de se prévaloir de leurs mérites, qu'il forme le souhait qu'ils se conduisent comme des évêques et des clercs dignes du nom qu'ils portent ${ }^{19}$.

Aux XI-XII siècles, l'inscriptio et l'intitulatio sont nécessairement prolongées d'une salutatio à la gloire du destinataire de l'épître; voir Bütow 1908, 62: «[...] Ferner braucht in der Salutatio nicht immer "salus" zu stehen, sondern es können dafür auch andere Wörter eintreten, insofern sie nur auf die Ehrung des Empfängers Bezug haben».

14. De ce point de vue, Malaterra témoigne d'une retenue plus grande que, par exemple, Guillaume de Jumièges ( "Gemmethicensis cenobita, omnium cenobitarum indignissimus Willelmus»; cf. van Houts 1992, 4) ou encore Guillaume de Malesbury («Willelmus bibliothecarius Malmesberiae»; cf. King 1998, 2).

15. Sur la place de Marthe et Marie dans la chronique, voir Oldoni 1977, 167, et D'Angelo 2003, 137 sq.

16. Une évolution similaire est rapportée en $\mathrm{IV} 7$ (p. 90,1-3) à propos du même personnage: Porro ille ecclesiam minus cultam, utpote a faucibus incredulae gentis erutam suscipiens, Marthae [iuris add. Pontieri] studiis primo studiosius inhaerens, breui ecclesiam omnibus necessariis prouehens, ad Mariae uices cum Martha exequendas transiit [...].

17. Cf. Lo Curto 2002, 23: "l'inizio di questo capoverso è incomprensibile per un probabile guasto del testo »; D’Angelo 2003, 139, évoque «il problema di mancanza di senso della frase».

18. L'expression est d'E. D'Angelo qui, de séquences arbitrairement définies, pose des conclusions précipitées : après avoir isolé une unité commune, frater Gaufredus, il place l'un vis-à-vis de l'autre deux morceaux de texte comportant chacun le nom Malaterra; si le premier donne un syntagme régulier ( $a b$ antecessoribus Malaterra agnomen trahens), le second emprunte à deux parties distinctes de la salutatio et ne constitue pas une unité signifiante (Malaterra cum assignatione nominis etiam). Voir D’Angelo 2003, 139 .

19. "[...] brother Geoffrey Malaterra, with his signature and his seal» (Wolf 2005, 42) est une interprétation extravagante, que le genre de la salutatio récuse absolument. 
On trouvera dans Lanham 1975 de nombreuses illustrations de cette construction, qui fut à l'origine de cette thèse. En voici quelques autres, présentées dans l'ordre chronologique, toutes tirées d'épîtres dédicatoires introduisant des ouvrages historiques $^{20}$ :

Domino in Christi gratia beatissimo atque in omni splendore sapientie perfectissimo Folcmaro archiepiscopo / Ruotgerus, seruorum suorum ultimus, / uere claritatis gloriam sempiternam (Ruotger de Cologne, Vita Brunonis archiepiscopi Coloniensis).

Gloriosissimo imperatori Heinrico tertio regi, ad pacem et bellum idoneo, / Wipo, Dei gratia presbyter, / quod seruus regalium seruorum huius orbis domino dominantium (Wipo [connu sous le nom de Wigberg], Gesta Chuonradi).

Pio, uictorioso atque orthodoxo summi Regis nutu Anglorum regi Willelmo / Gemmethicensis cenobita, omnium cenobitarum indignissimus, Willelmus / ad conterendos hostes Sansonis fortitudinem et ad discernendum iuditium Solomonis abyssum (Guillaume de Jumièges, Gesta Normannorum ducum [van Houts 1992, 4]).

Domino Seuero, Melnicensis ecclesie preposito, tam litterali scientia quam spirituali intelligentia predito, / Cosmas Pragensis ecclesie solo nomine decanus / post huius uite stadium in celesti regno brauium (Côme de Prague, Chronica Boemorum [Prologus ad Seuerum prepositum]).

Liberalium artium quibusque studiis pleniter imbuto et omnimode scientie sapientia delibuto archigeronte Geruasio / Cosmas quod dicitur haud dici dignus, Deo tamen et sancto Wencezlao famulantium famulus, / debite orationis munus et mutue dilectionis pignus (Côme de Prague, Chronica Boemorum [ad Geruasium magistrum prefacio]).

Domino amantissimo Rotberto filio regis Henrici et consuli Gloecestrensi / Willelmus bibliothecarius Malmesberiae / post emerita trophea in terris triumphare in caelis (Guillaume de Malmesbury, Historia Novella [Prologus (King 1998, 2)]).

Viris prudentissimis, pace et militia exercitatis, [domino] Vlrico et [domino] Heinrico, sacri palatii uni cancellario alteri notario, / Radewinus sanctae Frisingensis aecclesiae professione canonicus, ordine diaconus, licet indignus, / simul cum intellectu spiritu pietatis habundare (Radewin de Freising, Gesta Friderici I imperatoris [Pro$\log u s])$.

Vniuersis uniuersalis sanctae matris ecclesiae filiis in quorum manus libellus iste deuenerit / Ramensis ouilis coenobii pusillus grex/felici cursu aeternitatis brauium adipisci ([Historia Ramesiensis, sive] Liber benefactorum ecclesiae Ramesiensis (Ramsey Abbey) [Praefatio]).

Illustrissimo domno et benefactori suo precipuo Lupoldo, duci Austriae nobilisimo (sic), / Hermenger, domini patientia Christi pauperum seruus et fratrum sancti

20. Nous avons à chaque fois séparé d'un trait oblique les trois constituants de la salutatio. 
hospitalis Ierusalem prouisor humilis, cum uniuerso fratrum conuentu / debite orationis munus et sincere deuotionis instantiam (Historia de expeditione Friderici imperatoris).

Reuerendo patri et domino Ernaldo, abbati Rieuallis, / suus Willelmus, seruorum Christi minimus, / cum apparuerit Princeps pastorum, immarcescibilem percipere gloriae coronam (Guillaume Petit, dit de Newburgh [Yorkshire], Historia rerum Anglicarum).

Reuerentissimo domino et patri in Christo Huberto, Dei gratia Cantuariensi archiepiscopo, tocius Anglie primati, / unus ex minimis fratribus ordinis sancti Gileberti Sempinghamensis, / sanctorum meritis et premiis coequari (Prologus de uita sancti Gileberti confessoris).

Nobili uiro, domino suo carissimo, domino Engelberto, comiti de Marka, / Leuoldus de Northof, canonicus Leodiensis et abbas secularis ecclesie Visetensis Leodiensis dyocesis, / terram uestram et subditos cum Dei timore et iusticia fideliter gubernare (Levold de Northof, Chronica comitum de Marka).

\section{II}

(p. 3, 11-14) Sciendum tamen uobis est siue alteri quicumque libri huius recitator uel certe interpres accesseritis, $\langle\ldots$, , si... $>$ seriatim minus ordinate secundum tempora quibus facta sunt quae adnotantur uel certe aliqua obliuione praetergressa repereritis, non hoc iam mihi sed relatoribus culpando adscribatis [...]

sciendum $Z$ : - $\operatorname{dium} A \|$ accesseritis Desbordes :-rius $A$-rit $Z$, edd. $\|$ si add. edd. $\|$ ordinate $A Z$ : -ta Avenel D'Angelo $\|$ adnotantur $Z$ : adnoctantur $A \|$ hoc $A$ : haec $Z$, edd. $\| \operatorname{iam}\left(\operatorname{tam} Z\right.$ ut uid. $\left.{ }^{21}\right) \ldots$ sed $A Z: \operatorname{tam} .$. quam $e d d$. $\|$ adscribatis $A$ : -bantur $Z$, edd.

Le texte de Pontieri est défectueux, et son apparat, défaillant : il ne mentionne aucune variante! C'est pourquoi nous avons enregistré ci-dessus tous les éléments nouveaux ou utiles que la critique a apportés pour la constitution du texte.

Il est invraisemblable que les copistes n'aient sauté ici qu'un seul mot. Outre si, qui a été suppléé dans l'édition princeps - mais dont Pontieri ne dit rien, comme s'il l'avait lu également dans l'état de la tradition recueilli dans A -, et, comme M.-A. Avenel le note avec juste raison, la conjonction ${ }^{22}$ reliant la complétive (adscribatis) au verbe principal (sciendum est), il manque un morceau de texte constituant le

21. L'initiale de l'adverbe est assez singulièrement tracée pour autoriser les deux lectures. Sans doute faut-il lire avec Resta 1964, 48, iam. Indice concordant: un tam [...] sed, impossible, aurait suscité une correction de Zurita $\left(=\mathrm{Z}^{2}\right)$. Or celui-ci s'est borné à signaler le développement d'un At(tende) marginal.

22. Comparer p. $3,17[\ldots]$ sciendum est quoniam $[\ldots]$. La « construction paratactique $[\ldots]$ n'est pas habituelle chez Malaterra» (M.-A. Avenel, comm. ad loc.). 
pendant syntaxique d'aliqua $[. .$.$] praetergressa ^{23}$. À en croire le chroniqueur, son récit encourt deux critiques:

- le déroulement des faits ne respecte pas toujours l'ordre chronologique;

- la narration manque à l'exhaustivité, elle présente des lacunes.

L'exposé du premier grief est manifestement tronqué à son début. Deux syntagmes au moins font défaut : un pronom indéfini, correspondant à aliqua - car il est impensable que Malaterra se soit affranchi délibérément d'une des lois du genre historique ${ }^{24}$, et un participe à l'accusatif régi, comme praetergressa, par repereritis. M.-A. Avenel a paré à ce dernier manquement à la syntaxe en rétablissant ordinata, qu'elle tient pour « indispensable à la construction de la phrase dans l'état où elle a été transmise ${ }^{25}$. La faute supposée se retrouve, il est vrai, ailleurs; mais du fait de la lacune, rien n'interdit de penser que le participe attendu se lisait précisément dans le morceau de texte perdu, et qu'en conséquence, on ne doit pas se hâter de corriger les termes subsistants. On peut imaginer, à titre d'exemple, le supplément suivant: Sciendum tamen uobis est $[. .]<$. quoniam, si qua recensita $>$ seriatim $^{26}$ minus ordinate $[\ldots]$ repereritis, eqs. (= « [...] si vous trouvez que parfois, dans mon récit, la succession des événements n'est guère conforme au déroulement chronologique des faits rapportés, [...]») - le morceau de texte perdu occupant une ligne dans un modèle dont la mise en page était à deux colonnes.

\section{III}

I 14 (p. 15, 11-13) [...] Comes uero Humfredus, honestius ducens cum honore uitam finire quam cum dedecore [uita comite suscepto honore] priuari, [...] audacter hostibus occurrit $[\ldots]^{27}$

humfredus $C$ : umfridus $B$ om. $Z$, ed. pr. $\|$ honestius $C$ :-tus $Z$ om. $B$ potius post ducens supplens $\|$ occurrit $C Z$ : ac- $B$.

Depuis Pontieri, la critique, unanime, a estimé que ce chapitre présentait un passage interpolé, et les remèdes qu'elle préconise ne diffèrent entre eux que par le

23. La passivation du perfectum de praetergredior est attestée depuis Boèce, Eut. 8, 24: cf. P. Flobert, Les Verbes déponents latins des origines à Charlemagne, Paris, Société d'éditions «Les Belles Lettres » (Publications de la Sorbonne, Série «NS Recherches»; 17), 1975, p. 378.

24. Sur cette attention de Malaterra à la chronologie, cf. Desbordes 2005, 134 .

25. Voir «La nouvelle édition de la chronique de Geoffroi Malaterra», in Primo rapporto sull'edizione delle fonti del Mezzogiorno medievale (Atti del convegno internazionale, Napoli 11-12 aprile 2003) (à paraître).

26. Cette association de mots nous a été suggérée par les Gesta Normannorum ducum VII 1: Recensitis seriatim Rotberti magni ducis actibus eqs.

27. Nous avons débarrassé le texte cité des leçons que Pontieri devait soit à B (potius, suppléant honestius, omis; associés, ces adverbes font double emploi), soit à un sentiment incertain de la grammaire (sibi, qu'il a introduit motu proprio devant honestius, est une addition inutile). 
nombre des éléments réputés apocryphes. L'éditeur italien, considérant que la suite uita comite suscepto honore "non dà alcun senso ", l'exclut de son texte et la rejette dans l'apparat. M.-A. Avenel récupère uita, grammaticalement aisé à construire comme ablatif de séparation (uita [...] priuari), mais aussi parce qu'il est «donné par tous les manuscrits »-comme s'il en allait tout autrement pour la séquence intermédiaire, qu'elle aussi retranche, convenant après Pontieri qu'elle « ne fait pas sens ici» (comm. ad loc.). Nous-même, croyant à un saut régressif du même au même, nous avons proposé d'éliminer uita - réservant à suscepto honore le rôle que notre devancière avait attribué à ce terme indûment repris - et comite, qui ne s'accommodait pas à notre nouveau mot à $\operatorname{mot}^{28}$. Tout bien considéré, ces trois tentatives pour assainir le texte par des suppressions sont, à plus d'un titre, malvenues : améthodiques, puisqu'elles n'expliquent pas irréfutablement l'origine de l'intrusion présumée, elles ont en outre le tort de méconnaître une des sources implicites importantes des auteurs latins du Moyen Âge : la Vulgate. De fait, les mots censés irréductibles (uita comite) constituent une formule que Malaterra aura empruntée à la Bible ${ }^{29}$ :

- Gn 18, 10 Reuertens ueniam ad te tempore isto uita comite [...], ou

- Gn 18,14: iuxta condictum reuertar ad te hoc eodem tempore uita comite [... $]^{30}$. Il ne l'entendait pas dans l'acception qu'elle reçoit dans ces deux versets de l'Écriture - il ne s'agit plus du «temps de la vie », c'est-à-dire du «temps du renouveau », désignant la saison des pluies, «qui apportent la vie»-, mais dans celle que l'usage latin du mot comes ${ }^{31}$ avait imposée à la locution.

28. Cf. Desbordes 2002, 44 sq.

29. Sur une autre formule de la chronique restée longtemps inintelligible pour tous, alors qu'elle ne fait qu'adapter un passage scripturaire (ex sui cordis abundantia, I 6 [p. 10, 15]), voir Desbordes 2005, 122.

30. La même idée est exprimée par une proposition conditionnelle en $2 R 4,16$ : In tempore isto et in hac eadem hora, si uita comes fuerit, habebis in utero filium. Les exemples de cette construction personnelle sont plus nombreux que ceux de l'ablatif absolu: cf. Hier., epist. 119, 12; 125, 17 ; in Is. 63, 3-6 p. 748 ; Greg. M., epist. 9, 180 ; etc. M.-A. Avenel a attiré notre attention sur une variante de cette construction personnelle: en 2, 26 (p. 38, 40 sq.), les habitants de Gerace requièrent de Guiscard qu'il leur jure que, si, sa liberté recouvrée, il réchappe du sort auquel son frère Roger le destine, il n'élèvera pas de château fort, sa vie durant, dans leurs murs (dum sibi uita comes fuerit [= "aussi longtemps qu'il demeurera en vie»]). Sur l'expression concurrente si uita suppetet, qui «constitue un lieu commun aussi banal en latin que l'expression "si Dieu me prête vie" en français ", voir F. Paschoud, Histoire Auguste V ${ }^{\text {re }}$ partie, Vies d'Aurélien, Tacite, Paris, Les Belles Lettres (CUF), 1996, p. 142.

31. Sur cet emploi particulier de comes, «de re incorporea», dont nos dictionnaires classiques ne font guère état, cf. TLL III 1775,84-1776,6 (Bannier, 1911), citant Sidon., epist. 9, 16, 3 (v. 16 : comite arte); Drac., satisf. 202 (non habet haec [sc. clementia] comitem participemque negat); Greg. Tvr., uit. patr. 20, 2 (oratione comite). On peut comparer à notre passage cet extrait d'une des lettres qui servent de préface aux Étymologies d'Isidore: [...] De cetero peto ut, dum uita comite portitori ad nos regredi fuerit oportunitas, uestris nos iubeatis laetificare eloquiis (orig., praef. epist. B [ed. Lindsay]). 


\section{Emendatiunculae Malaterrenae}

Contrairement à ce qu'on a pu écrire, le texte transmis est correct pour la grammaire comme pour le sens : «[...] estimant qu'il était plus honorable de perdre la vie avec honneur que de continuer de vivre, dépossédé ignominieusement de la dignité qu'il avait revêtue [...] » [litt ${ }^{\mathrm{t}}$ : que d'être dépossédé dans l'ignominie, la vie étant toujours sa compagne (uita comite), de la dignité qu'il avait revêtue].

Quant aux conjectures malheureuses que ce morceau de texte, mal compris, aura occasionnées, elles ne méritent pas d'être signalées dans un apparat.

Olivier Desbordes

Université de Caen Basse-Normandie

\section{Références bibliographiques}

Biblia Sacra iuxta vulgatam versionem adiuvantibus Bonifatio Fisher OSB, Johanne Gribomont OSB, H.F.D. Sparks, W. Thiele recensuit et brevi apparatu instruxit Robertus Weber OSB. Editio tertia emendata [...], Stuttgart, Deutsche Bibelgesellschaft, 1983 ( $1^{\text {re }}$ éd. 1969).

BüTow A. (1908), Die Entwicklung der mittelalterlichen Briefsteller bis zur Mitte des 12. Jahrhunderts, mit besonderer Berücksichtigung der Theorieen der ars dictandi, Greifswald, Buchdruckerei Hans Adler (Inaug.-Diss.).

D’Angelo E. (2003), «Un “doppio” prologo al “De rebus gestis Roberti et Rogerii” di Goffredo Malaterra? ", in Storiografi e cronologi latini del Mezzogiorno normanno-svevo, Naples, Liguori Editore (Nuovo Medioevo ; 69), p. 134-142.

Desbordes O. (2002), "Notes de critique textuelle sur le livre premier de Geoffroi Malaterra », in Mélanges Pierre Bouet. Recueil d'études en hommage à Pierre Bouet, F. Neveux, C. Bougy (éd.), Caen, Annales de Normandie (Cahier des Annales de Normandie ; 32), p. 39-47.

Desbordes O. (2005), « Nouvelles notes critiques sur les deux premiers livres de la chronique de Geoffroi Malaterra », Kentron, 21, p. 111-159.

Geoffrey Malaterra (Wolf 2005), The Deeds of Count Roger of Calabria and Sicily and of his Brother Duke Robert Guiscard, K. B. Wolf (trad.), Ann Arbor, The University of Michigan Press.

Geoffroi Malaterra (Pontieri 1927-1928), De rebus gestis Rogerii Calabriae et Siciliae Comitis et Roberti Guiscardi Ducis fratris eius auctore Gaufredo Malaterra monacho Benedictino, E. Pontieri (éd.), Bologne, N. Zanichelli (Rerum Italicarum Scriptores, Raccolta degli storici italiani dal cinquecento al millecinquecento ordinata da L.A. Muratori, t. V-1, fasc. 1; 2-3).

Goffredo Malaterra (Lo Curto 2002), Ruggero I e Roberto il Guiscardo, V. Lo Curto (éd. et trad.), Cassino, Francesco Ciolfi Editore. 


\section{Olivier Desbordes}

Lanнам C.D. (1975), Salutatio Formulas in Latin Letters to 1200 : Syntax, Style, and Theory, Munich, Arbeo Gesellschaft (Münchener Beiträge zur Mediävistik und RenaissanceForschung; 22).

Lucas-Avenel M.-A. (2001), Édition critique avec traduction et commentaire des deux premiers livres de la chronique de Geoffroi Malaterra, thèse de doctorat (Langues et littératures anciennes) sous la direction de L. Callebat et S. Fodale, Université de Caen Basse-Normandie, 4 fasc. (I-CXLVIII ; 140 p., table des matières; 149-288 p.; 289-458 p.; 9 hors-texte) (dactyl.).

Oldoni M. (1977), « Mentalità ed evoluzione della storiografia normanna fra l'XI e il XII secolo in Italia », in Ruggero il Gran Conte e l'inizio dello stato normanno (Atti delle seconde giornate normanno-sveve, Bari, 19-21 maggio 1975), Rome, Centro di ricerca pergamene medievali e protocolli notarili (Fonti e studi del Corpus membranarum italicarum ; 12) (réimpression: Bari, Edizioni Dedalo [Centro di studi normanno-svevi, Università degli studi di Bari ; 2], 1991), p. 143-178.

Resta G. (1964), «Per il testo di Malaterra e di altre cronache meridionali », in Studi per il CL anno del Liceo-Ginnasio «T. Campanella» (1814-1964), Reggio Calabria, p. 3-6o.

Simon G. (1958; 1959-1960), «Untersuchungen zur Topik der Widmungsbriefe mittelalterlicher Geschichtsschreiber bis zum Ende des 12. Jahrhunderts ", Archiv für Diplomatik, Schriftgeschichte, Siegel-und Wappenkunde, 4, p. 52-119 (Erster Teil) ; 5/ 6, p. 73-153 (Zweiter Teil).

William of Jumièges (van Houts 1992), The Gesta Normannorum Ducum of William of Jumièges, Orderic Vitalis, and Robert de Torigni, E.M.C. van Houts (éd. et trad.), Oxford, Clarendon Press (Oxford Medieval Texts), 2 vol.

William of Malmesbury (King 1998), Historia Novella. The Contemporary History, E. King (éd.), K.R. Potter (trad.), Oxford, Clarendon Press (Oxford Medieval Texts). 